\title{
Case Report: Differential Diagnosis of a Complex Postpartum Patient with Cardiac Symptoms
}

\section{Cody Thompson, PT, DPT, GCS}

Assistant Professor, Department of Physical Therapy, Alabama State University, 334-229-5615, United States.

\author{
Article Details \\ Article Type: Case Report \\ Received date: $11^{\text {th }}$ February, 2020 \\ Accepted date: $14^{\text {th }}$ March, 2020 \\ Published date: $18^{\text {th }}$ March, 2020
}

"Corresponding Author: Cody Thompson, Assistant Professor, Department of Physical Therapy, Alabama State University, 334-229-5615, United States. E-mail: cthompson@alasu.edu

Citation: Thompson, C. (2020). Case report: Differential diagnosis of a complex postpartumpatient with cardiac symptoms. J Rehab Pract Res 1(1):101. https://doi.org/10.33790/jrpr1100101

Copyright: $\left({ }_{2} 2020\right.$, This is an open-access article distributed under the terms of the Creative Commons Attribution License 4.0, which permits unrestricted use, distribution, and reproduction in any medium, provided the original author and source are credited.

\begin{abstract}
s
Background: This case report describes the physical therapy differential diagnostic and clinical decision-making processes utilized in the management of a complex post-partum patient. This case is significant in that it demonstrates how multiple physical and physiological systems can overlap with a mechanical abnormality that would often be difficult to differentially diagnosis.
\end{abstract}

Case presentation: A 31-year-old female patient with an initial presentation of shoulder and thoracic pain that progressed into symptoms similar to those of myocardial infarction (MI). She was initially medically diagnosed with Gastroesophageal Reflux Disease (GERD) and anxiety, though symptoms were unresolved after addressing this with positional and dietary changes. Medication was refused. After multiple events, imaging, testing, and further systems review, the symptoms she described and the objective findings led her clinical team to determine she suffered from vagal nerve entrapment at the esophageal hiatus, which was successfully treated by the physical therapist in conjunction with chiropractic care.

Discussion: Over a period of 23 days and 5 physical therapy sessions, a visit to the local emergency department, a visit to the patient's primary care physician a visit to a cardiologist, and an evaluation by a chiropractor, a diagnosis and treatment plan was developed and carried out. The patient management approach included examination (subjective and physical), evaluation of findings, medical and physical therapy diagnoses, prognosis, plan of care, intervention, and evaluation of outcomes.

Conclusion: This case report demonstrates the need for physical therapists to incorporate a comprehensive patient management approach in order to efficiently maximize patient outcomes, and also places the challenge on physical therapists to engage in interprofessional care for differential diagnosis, imaging, and expertise outside the PT/PTA scope of practice. It also demonstrates the need for physical therapists to be more aware of overlapping physical, mechanical and physiological systems during evaluation.

Keywords: Physical Therapy, Hiatal Hernia, Vagus Nerve Entrapment, Differential Diagnosis, Clinical Decision-making

\section{Background}

The American Physical Therapy Association (APTA) describes physical therapy diagnosis as a process used to label and identify the impact that a condition has on function. The physical therapy diagnostic process is driven by clinical decision-making (CDM) including physical therapy scope of practice considerations. In this case report, the APTA patient management CDM model was utilized [1].The APTA patient management CDM model includes examination (subjective and physical), evaluation, physical therapy diagnosis, prognosis, plan of care, intervention, and evaluation of outcomes [1]. The subjective examination includes a detailed patient history and pertinent questions intended to facilitate a physical therapy diagnostic hypothesis. Subsequently, specific physical examinations are performed and evaluated to support or refute the physical therapy diagnostic hypothesis. Periodically, clinicians determine that a patient is not appropriate for physical therapy and further medical consultation is warranted by alternative inter-disciplinary providers [2]. Otherwise, the clinician reaches a physical therapy diagnosis, determines prognosis, and generates a plan of care. The intervention is implemented and outcome measures are obtained during each session.

The plan of care for patients demonstrating cardiac involvement who have not seen a physician prior to physical therapy are often referred to a physician for differential diagnosis, specifically for safety and liability purposes. Patients with cardiac involvement can be challenging for physical therapists to manage because of the inherent complexity of the condition; this is compounded in the cases of post-partum, nursing females due to difficulty maintaining homeostasis [4]. Many complex patients are referred to physical therapy with non-specific diagnoses and the responsibility is placed on the physical therapist to determine the origin of symptoms in order to decide the appropriate plan of care. Therefore, the purpose of this case report was to describe the CDM process utilized during the management of a complex cardiopulmonary patient with multiple events and triggers. The patient was selected because of the non-specific medical diagnosis provided by the physician as well as the need for continual re-examination and physical therapy differential diagnosis throughout the patient management process due to continued symptom production, as well as the need for interprofessional and interdisciplinary involvement.

\section{Case presentation}

\section{Subjective examination}

The patient was a 31-year-old female post-partum mother of five children who experienced insidious, gradual onset of chest tightness, heart palpitations and arrhythmias, shortness of breath, weakness, fatigue, dizziness, thoracic pain and cervical pain. She had initially been seen by her primary care physician (PCP) for her cardiac discomfort and fatigue. The physician found no bloodwork abnormalities, and referred the patient for an echocardiogram (ECG) to rule in or rule out a diagnosis of Mitral Valve Prolapse (MVP) or 
Mitral Valve Regurgitation (MVR). ECG findings were normal with no MVP/MVR noted.

Two days later, the patient was complaining of feeling nearsyncopal, severely fatigued, an inability to function at her normal, generalized weakness, shortness of breath, and the heart palpitations which she described as a "flip-flopping inside my chest". She also had described her chest tightness as that of "an elephant sitting on my chest". A family member drove her to the emergency department (ED) when symptoms became life-altering and alarming. Her previous level of function was as a stay-at-home mother, tending to the daily needs of herself, her husband and her five children. The ED physician made an initial medical diagnosis of arrhythmia, GERD and anxiety-related fatigue. EKG produced several abnormal atrial events related to depolarization and repolarization (per EKG strip), though this was not noteworthy to the attending ED medical staff while looking at a six-second strip of the EKG results. The patient was discharged with a prescription of Xanax to be taken as needed and to follow-up with her primary care physician, which the patient refused because she was currently nursing her most recent child and did not want any interference of medication in her breastmilk. She was also encouraged to rest as much as possible. The patient's spouse was a physical therapist and he contacted the primary author requesting a physical therapy consultation for further differential diagnosis and treatment planning. Because of the severity and frequent nature of her symptoms as noted above, and her inability to carryout normal ADLs for herself and in caring for her five young children, a family member drove her to the appointment.

The physical therapy consultation occurred two days after the initial onset of symptoms. The patient's chief complaints included insidious, gradual onset of chest tightness, heart palpitations and arrhythmias, shortness of breath, weakness, fatigue, dizziness, thoracic pain and cervical pain. The patient'spast medical history was unremarkable, though her familial medical history includes various autoimmune disorders, paternal CABG at the age of 50, anxiety, depression, and GERD. Current symptoms of cervicothoracic pain ranged from 3-7/10, as per the visual analog scale (VAS), and her current cervicothoracic pain VAS was 5/10. The patient was taking no medications. No co-morbidities were reported, though she has five children and her most recent birth occurred thirteen months ago. She states that she has not felt that her body has healed properly after this most recent pregnancy. The patient goals included resolving her pain and fatigue, gaining strength, and most importantly resolving the cardiopulmonary compromise and discomfort she is suffering.

\section{Physical examination}

The results of cervicothoracic and thoracolumbar spinal mobility showed moderate anterior to posterior hypermobility between the T5 and T9 segments, with hypomobility noted at the cervical and lumbar spines. Neurological medical screening examinations (dermatomal and myotomal examinations) were unremarkable. The patient demonstrated point tenderness at the $\mathrm{T} 7$ spinous process, and at the ligamentous space where ligamentum flavum is located between T7-T8. Resting pain at these areas was at 3/10, with increase to $8 / 10$ with palpation. Postural examination showed mildly rounded shoulders bilaterally and upper cross syndrome present.The patient has complained of occasional tension headaches. LE and UE MMT were grossly $4 / 5$ bilaterally, though periscapular MMT equaled $3+/ 5$ bilaterally. Resting heart rate (RHR) ranged between $60 \mathrm{bpm}$ and $72 \mathrm{bpm}$ upon regular, intermittent evaluation. Radial pulses noted frequent arrhythmias which were also noted with auscultation. Chest and posterior thoracic wall (cardiopulmonary) auscultation showed clear lung sounds, no cardiac rubs, no pulmonary wheezes, absence of painful cough and cough was unproductive. No signs of infection, and the patient was afebrile. Chest tightness, discomfort and SOB increased with supine positioning, and was alleviated from sitting and standing, with forward lean on elbows. Additionally, patient described occasional nausea with onset of cardiac arrhythmias and palpitations.

\section{Pericarditis Lean Test}

Purpose: assessment for pericarditis

Procedure: patient seated/standing leaning on elbows at a 45-90 degree lumbopelvic flexion; this places the cardiac and surrounding tissues in a dependent position alleviating stress from the pericardial membrane

Sensitivity and Specificity: Not Established

\begin{tabular}{|l|l|l|l|}
\hline Examination & Provider & Results & Diagnosis \\
\hline Vital Signs & PT/ED & WNL, arrhythmia noted with RHR & N/A \\
\hline EKG & MD & Arrhythmia & GERD, Anxiety \\
\hline ECG & DO & No structural abnormalities & N/A \\
\hline Holter Monitor & Cardiologist & Arrhythmia & N/A \\
\hline Pericarditis Repositioning Test & PT & $(+)$ & $\begin{array}{l}\text { Undecided pericarditis pending } \\
\text { positional diagnosis }\end{array}$ \\
\hline CBC & ED & WNL & N/A \\
\hline Hormone Panel & DO & WNL & N/A \\
\hline Chest X-ray & ED & negative & N/A \\
\hline Doppler & MD & negative & N/A \\
\hline Lung Auscultation & PT & negative & N/A \\
\hline Heart Auscultation & PT/ED & arrthymia & N/A \\
\hline Thoracic A/P Motion & PT & $\begin{array}{l}\text { Hypermobile at T5-T9 (relative to } \\
\text { surrounding segments) }\end{array}$ & N/A \\
\hline Spinal X-ray & Osteophytes at T7-T8 & Osteoarthritis \\
\hline Pericarditis Lean Test & PT & Resolution of cardiac discomfort & $\begin{array}{l}\text { Positional involvement of cardiac/neural } \\
\text { tissue }\end{array}$ \\
\hline
\end{tabular}

Table 1. General Description of Physical Examinations Utilized in Case Report. 


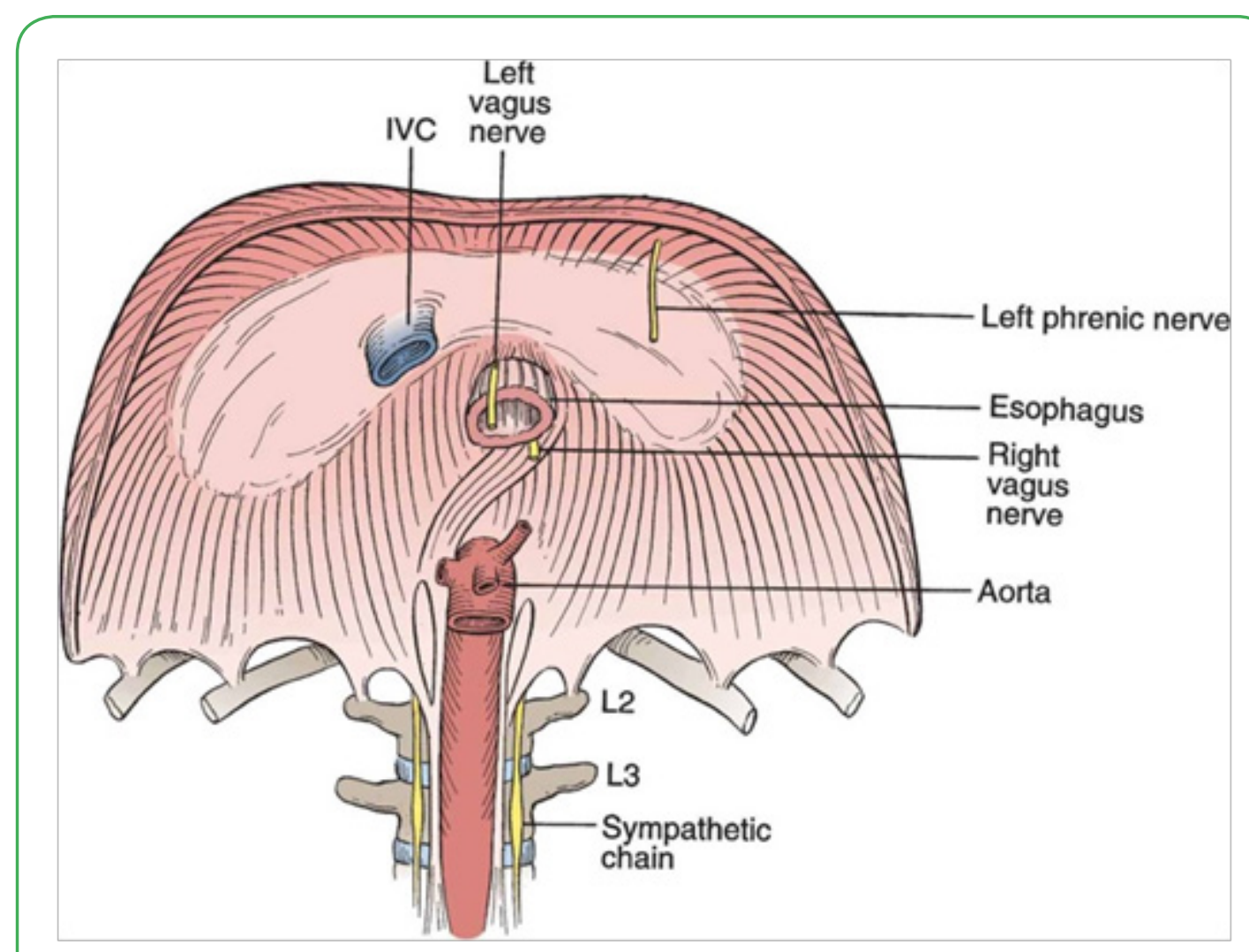

Figure 1 demonstrating the anatomical location of the left and right vagus nerves in relation to hiatal hernia.

\section{Intervention}

The patient was seen for a total of 6 sessions over a period of 23 days.

Session 1-(Day 1) The patient was seen by a physical therapist (PT) for assessment and PT diagnosis. Based on the presentation of resolution of symptoms with anterior lean (Pericarditis Lean Test), and the patient's history of hiatal hernia, GERD and multiple pregnancies, it was believed that the patient was presenting with an entrapment of the Vagus nerve as it entered and exited the esophageal hiatus. PT treatment began with postural training exercises, anterior cervical strengthening exercises and periscapular strengthening. The patient was given isometric chin tucks, isometric scapular squeezes (10 x 10 second holds for each, 2x/day) and pectoralis major/minor stretches to begin postural correction ( $5 \times 15$ seconds, $2 \times /$ day). Because the patient also self-described a weak pelvic floor, she was also prescribed Kegel exercises twice daily in combination with deep breathing, to increase intra-abdominal pressure, producing a forced isometric of the perineal muscles. She also has a two-finger width diastasis recti from her second pregnancy; these exercises were given verbally and as digital pictures for home exercise program.

Session 2-(Day 2) The patient was seen by a local chiropractor(DC) for spinal and visceral assessment after she had become frustrated with "inadequate holistic care" provided by her physicians and the ED. The chiropractor took x-ray imaging of the entire body, performed a postural assessment and examined her abdomen. The chiropractor then performed spinal manipulations of T2-T6 and a counter-nutated sacroiliac joint on the right side. He also noted (via palpation) an elevated cardia at the esophageal-stomach junction, which he manually massaged caudally. This treatment provided initial, temporary resolution of her cardiac symptoms.

Session 3-(4 days after initial consultation by PT). The patient was seen by her physical therapist and her reported cardiac symptoms had improved to a VAS range of 3-6/10 and she had felt a slight increase in her energy level, while experiencing less frequent cardiac discomfort. She continued to complain of suboccipital headaches and T7-T9 tenderness at $4 / 10$ on a VAS. Her chief complaint at this time remained occasional cardiac discomfort headaches, and continued weakness/fatigue below her prior level of function.

Upper cross syndrome still presents, with 2.5 inches of height from plinth on the left side and 3 inches of height on the right shoulder when performing the Pec Minor Length Test. Focus of her second PT session is improving postural abnormalities through anterior chest wall stretching, postural thorax wall and periscapular strengthening, and gait training with mirror feedback for improved posture during gait.

Session 4-(5 days after initial consultation) The patient reported to the PT that her cardiac symptoms had improved to a VAS range of 2-4/10 and home program was going well. Her chief complaint at this time remained occasional paroxysmal cardiac and/or arrhythmic events that usually were coming on at the end of the day as she was tired and occasional suboccipital= headaches. Physical re-examination of the head, neck and shoulder girdles showed continued mildly forward headand upper cross syndrome. The intervention consisted of home program progression and manual pec minor myofascial release and corner stretching. The patient was instructed to perform 3 sets of standing corner pec major stretching 5 x 20 seconds each. Isometric rows for rhomboid and middle trapezius strengthening were added to the home exercise program. She was also placed on a basic cardio regimen while remaining asymptomatic to improve the patient's activity tolerance, but also to improve her breathing and LE/pelvic strength. The patient tolerated this well. Communication via e-mail was planned for after the physician visit.

Session 5 - (8 days after initial consultation) The patient reported to her DC that symptoms had improved to a VAS range of 2-4/10 and home program was going well. The chiropractor focused on improving mobility of her lumbopelvic region via rotational spinal manipulation, as well as continued caudal esophageal massage. DC assessment shows near resolution of the esophageal/hiatal hernia, and these findings align with resolution of her cardiac symptoms. 


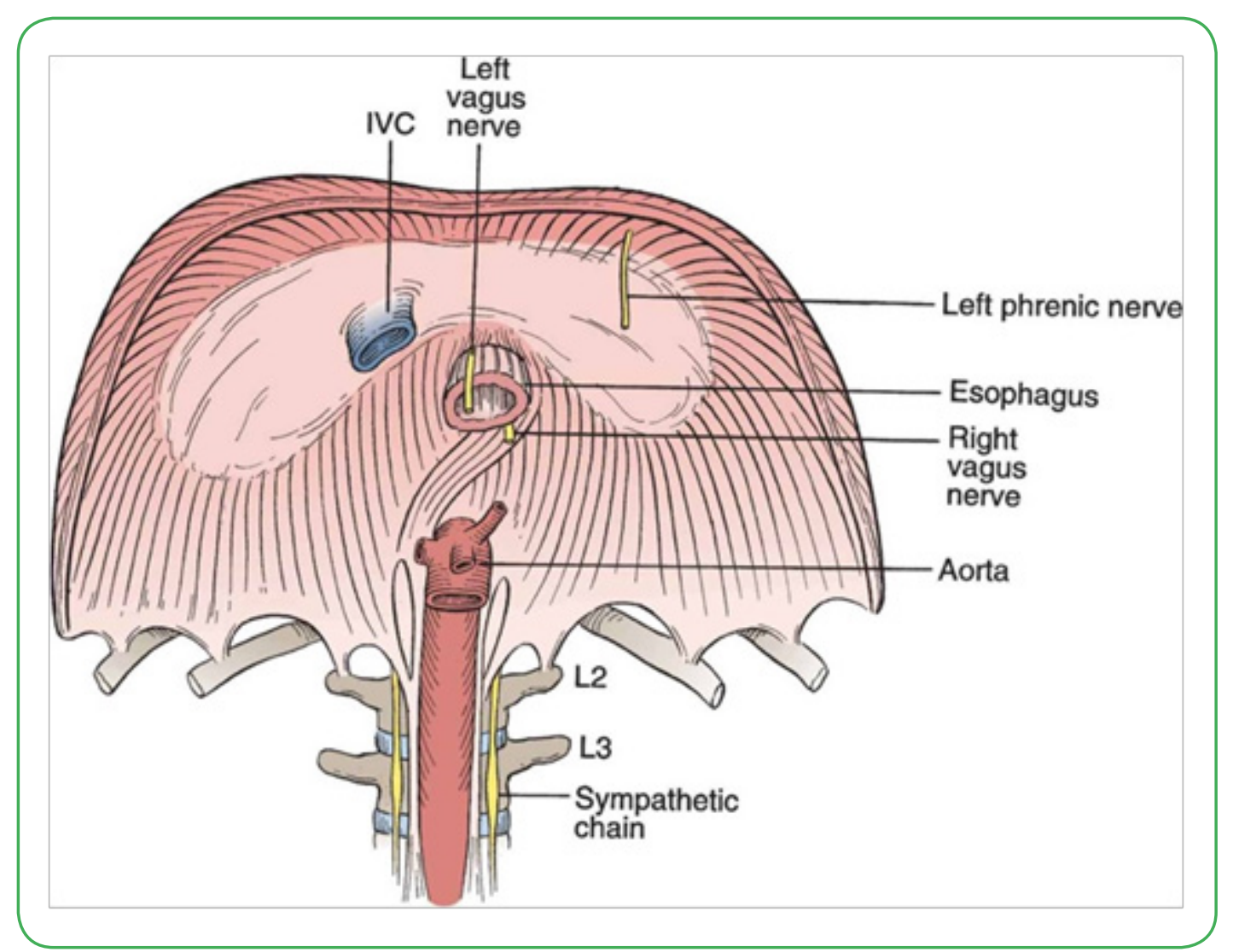

Session 6 - (12 days after initial consultation) The patient reported to her PT/DC that her cardiac symptoms had improved to a VAS range of $0 / 10$ and home program was going well. Her chief complaint at this time remained occasional discomfort to palpation or lying down, at the T7-T9 vertebrae. The intervention consisted of home program progression and postural education.

\section{E-Mail Consultation-(15 days after initial consultation)}

The patient reported she had been returning to daily activities without heart palpitationsor cardiac discomfort, and stated that "The weakness and fatigue is still there a bit, but that is mostly because of lack of sleep from the baby waking." The patient's chiropractor also confirmed that after three bouts of esophageal massage and thoracic spinal manipulation to address her hiatal hernia which caused the vagus nerve entrapment, the hiatal hernia is resolved.

\begin{tabular}{|l|l|l|}
\hline Intervention & Provider & Results \\
\hline $\begin{array}{l}\text { Postural Re-training in combination with deep } \\
\text { breathing techniques }\end{array}$ & PT & $\begin{array}{l}\text { Minimal results alone; immediate results with esophageal caudal } \\
\text { massage during deep breathing }\end{array}$ \\
\hline $\begin{array}{l}\text { Periscapular Strengthening; Anterior Cervical } \\
\text { Strengthening }\end{array}$ & PT & Progressive improvement \\
\hline Thoracic spinal manipulations/mobilization & PT/DC & $\begin{array}{l}\text { Intermittent resolution of thoracic pain, little to no resolution of cardiac } \\
\text { symptoms }\end{array}$ \\
\hline Esophageal/Epigastric Massage & DC & Resolution of symptoms \\
\hline Xanax & MD & Refused medications \\
\hline
\end{tabular}

\section{Discussion}

This case report describes the physical therapy differential diagnosis and clinical decision-making process with a complex patient. In some cases, physical therapy diagnosis includes the pursuit of pathological origin and may take into account information determined from other interdisciplinary healthcare providers. For example, many patients are referred to physical therapy with a medical diagnosis of "generalized weakness", which is a symptom rather than pathology. In order to provide the appropriate clinical intervention, physical therapy examination and evaluation are performed to determine the origin of the weakness. In the present case, successful and efficient management of this patient scenario was dependent upon knowing specific systems involvement, diagnostic testing necessary for objective findings, available healthcare providers in the area, and how certain symptoms and their timing and provocation are related back to certain physiological systems and physical structure.

Re-examination was important as the primary diagnosis changed over the course of physical therapy management and new physical therapy diagnoses were made. The physical therapy diagnostic process is driven by CDM and requires determination of whether or not a patient's clinical presentation is within the scope of physical therapy practice. Schenkman et al., [2] report that CDM frameworks are "instrumental in guiding clinicians through a comprehensive process of patient management, communicating with other healthcare providers and facilitating the educational process." This author believes that this statement embodies the patient situation describes here quite accurately.

Boissonnault and Ross [3] identified 78 published case reports where 
physical therapists referred patients to physicians and subsequent medical diagnoses were made. The authors stated that "an important role for physical therapists in the healthcare delivery system is to recognize when patient referral to a physician or other healthcare provider is indicated." The APTA patient management CDM framework is flexible enough in its format that clinicians can incorporate aspects of multiple CDM models on a case-by-case basis [1].

\section{Conclusion}

This case report illustrates the physical therapy differential diagnosis and clinical decision-making processes used with a complex patient. The subjective examination was used to generate a diagnostic hypothesis and specific physical examinations were performed and evaluated to support it. Subjective information and objective testing helped to determine what interdisciplinary care may need to be involved other than PT. Once the physical therapy diagnosis was made, prognosis and plan of care were determined. Appropriate interventions were implemented, interdisciplinary providers were involved, and the patient was re-examined each session. This comprehensive physical therapy patient management approach led to an efficient and positive treatment outcome.

\section{List of abbreviations}

APTA: The American Physical Therapy Association

CDM: Clinical decision-making

HOAC: The hypothesis-oriented algorithm for clinicians

ED: Emergency department

VAS: Visual analog scale

ECG: Echocardiogram

EKG: Electrocardiogram

DO: Osteopathic physician

DC: Chiropractor
Conflicts of Interest: The authors declare no conflicts of interest.

\section{References}

1. Guide to Physical Therapist Practice. (2001). Second Edition. American Physical Therapy Association. Phys Ther. 81:9-746.

2. Schenkman, M., Deutsch, J.E., \& Gill-Body, K.M. (2006). An integrated framework for decision making in neurologic physical therapist practice. Phys Ther 86:1681-702.

3. Boissonnault, W.G., \& Ross, M.D. (2012). Physical therapists referring patients to physicians: a review of case reports and series. J Orthop Sports Phys Ther 42:446-54.

4. Masiukiewicz, U. S., \& Insogna, K. L. (2004). Calcium Homeostasis and Disorders of Calcium Metabolism During Pregnancy and Lactation. Medical Complications During Pregnancy, 181-191. doi: 10.1016/b978-0-7216-0435-0.500134 